\title{
El cuidado en el hogar a los enfermos crónicos: un sistema de autoatención
}

\author{
Home care for the chronically ill: \\ a self-care health system
}

Leticia Robles Silva 1

\section{Centro Universitario de Ciencias de la Salud, Departamento de Salud Pública, Universidad de Guadalajara, Jalisco, México. \\ Correspondencia Leticia Robles Silva Universidad de Guadalajara. Pirineos \# 1462, Col. Independencia CP 44340, Guadalajara, Jalisco, México. lrobles@cucs.udg.mx}

\begin{abstract}
This article focuses on home care for chronically ill adults and seniors. According to our thesis, home care should be understood as a self-care system, and its aim is to guarantee the individual's social and bodily survival. Home care consists of three areas, related to illness, the home, and to life history. Caregiving, usually under women's responsibility, is present throughout the history of the illness and the health-seeking process. The article analyzes these issues in light of the ageing process, the epidemiological changes occurring worldwide, and the urgency to incorporate this analysis into the heath care research agenda.
\end{abstract}

Caregivers; Chronic Disease; Family; Patients

\section{Introducción}

Una de las corrientes que gana mayor terreno en la discusión de la atención a la enfermedad es el auto-cuidado y el cuidado brindado en el hogar, sobre todo este último en relación con los enfermos crónicos y los ancianos. El cuidado es un término que se ha introducido en el lenguaje de terapeutas y trabajadores de la salud como una especie de recurso ilimitado para enfrentar las demandas de atención de este tipo de enfermos, pero que poca reflexión ha generado acerca del sitio que ocupa entre los vastos sistemas médicos existentes en la sociedad.

El presente trabajo reflexiona acerca de la pertinencia de ubicar el fenómeno del cuidado en el hogar a enfermos crónicos como parte del sistema de autoatención. El planteamiento central es que el cuidado en el hogar es un proceso diferente al de la automedicación, al incluir acciones más amplias que este último. Más que ser conceptualizado como una variante de éste, el cuidado es un proceso diferente. La presente reflexión teórica deriva tanto de lo reportado en la literatura sobre el cuidado como de los resultados de una investigación etnográfica sobre el cuidado que realicé entre 1997 y 2001 en un barrio popular de Guadalajara, México, sobre el cuidado a enfermos crónicos. Los informantes fueron nueve mujeres y nueve hombres adultos, ancianos enfermos crónicos y sus cuidadores. Siete hombres y 19 mujeres, tam- 
bién adultos y ancianos, eran quienes los cuidaban.

El trabajo se divide en cuatro partes. Primero, se ubica temáticamente la autoatención, el tipo de conocimiento generado y la pertinencia de abrir la discusión a otros procesos. La segunda parte sitúa el cuidado como parte de un proceso más amplio de atención a los enfermos y al mismo tiempo se argumenta sobre la importancia de su estudio. La tercera parte desarrolla las nociones y los argumentos para conceptualizar al cuidado como un proceso de autoatención. Finalmente, se discuten algunas implicaciones empíricas de una propuesta de esta naturaleza.

\section{Algunos antecedentes teóricos: los sistemas médicos}

Hoy día se reconoce la existencia de una pluralidad de sistemas de atención a la enfermedad en cualquier sociedad o comunidad del mundo. Dichos sistemas son la biomedicina, las medicinas alternativas y la autoatención, presentes en mayor o menor medida en cualquier contexto espacial como respuesta diagnóstica y terapéutica a la enfermedad.

La descripción y explicación de los tres sistemas han sido objeto de la sociología y la antropología médica, pero con un especial énfasis en la biomedicina y las medicinas alternativas. Kleinman 1 y Menéndez 2 afirmaban hace más de diez años que poca investigación existía sobre el funcionamiento de la autoatención durante los años previos a la década de 1980. El panorama no cambió substancialmente durante los siguientes años. Esta ausencia de interés por el tema resulta paradójico por que teóricamente se sostiene que la autoatención "primer nivel real de atención a la enfermedad" 2,3 o que es "la parte central del sistema de atención a la enfermedad" 1 (p. 50).

De acuerdo a lo investigado, el componente más analizado de la autoatención es la automedicación, que se refiere esencialmente a la prescripción y uso de medicamentos, terapéuticas coadyuvantes como masajes, y terapias tradicionales como infusiones o cataplasmas. Sabemos que el hogar es el espacio por excelencia de la automedicación, ahí se realiza el diagnóstico de la enfermedad, la selección, prescripción y administración de medicamentos o terapias, todo antes de acudir a los terapeutas. Es parte de la "carrera del enfermo" en su fase inicial, sobre todo en el momento del diagnóstico; y la misma es realizada por la mujer. La automedicación es ampliamente estudiada en torno a las enfermedades infectocontagiosas y/o la salud materno-infantil.

Esta tendencia de estudiar la autoatención centrada en la automedicación debe ampliarse frente a las nuevas condiciones sociales y epidemiológicas. Tres puntos lo justificarían.

Uno, el casi olvido de otros miembros enfermos de la familia. Tradicionalmente, los niños y sus madres eran los sujetos de indagación, lo que respondía a una situación demográfica y epidemiológica: los niños eran la mayor proporción de la población en la primera mitad del siglo XX y quienes más enfermaban y morían. Condición que se modificó en el último tercio del siglo pasado cuando aumenta significativamente la población anciana. Realidad demográfica que permanecerá durante el siglo XXI 4, cambiando la composición de enfermos al interior de las actuales familias. Ahora, enferman tanto niños como ancianos.

Dos, la exclusión de otras enfermedades prevalecientes en el panorama epidemiológico. Las enfermedades infecto-contagiosas fueron el rasgo de la primera mitad del siglo XX; hoy, las enfermedades crónicas son el principal componente del perfil epidemiológico de casi cualquier nación y lo seguirán siendo durante el siglo XXI 5.

Tres, más allá de la automedicación no se han desarrollado ni indagado otros procesos de autoatención. Poco o nada sabemos acerca de lo que acontece con la autoatención cuando se involucran otro tipo de acciones no vinculadas, directa o indirectamente, a la curación o limitación a la enfermedad.

Frente al conocimiento generado hasta hoy día y ante los cambios en el panorama demográfico y epidemiológico es indispensable abrir la discusión hacia nuevos campos de indagación que analicen fenómenos no vistos hasta ahora. Si queremos explicar las nuevas condiciones de salud/enfermedad y su atención en el interior del hogar requerimos de construir nuevos conceptos que enriquezcan el análisis del proceso de autoatención. La propuesta es considerar el cuidado en el hogar a los enfermos crónicos como parte del sistema de autoatención. 


\section{Un tema emergente: el cuidado en el hogar a los enfermos crónicos adultos y ancianos}

El cuidado en el hogar a enfermos crónicos adultos y ancianos, o cuidado informal como algunos autores lo nombran 6,7, es un tema privilegiado en los estudios antropológicos y sociológicos de la vejez. La creciente literatura da cuenta de la importancia del tema, de acuerdo a Pearlin \& Zarit 8 el estudio del cuidado, que se inició en 1970, está en una etapa de consolidación después de 15 años de investigación. Los estudios abundan con respecto al cuidado a ancianos y enfermos crónicos de áreas urbanas en países anglosajones; siendo muy reciente la investigación en países como México, Puerto Rico, Brasil y España.

El cuidado es un tema ineludible de la agenda de investigación actual. El envejecimiento y las enfermedades crónicas originan discapacidades que afectan la autonomía del individuo dando paso a un mayor número de adultos y ancianos dependientes de la atención y el cuidado de los otros. Ambos fenómenos han permitido la visibilidad del cuidado. Esta visibilidad no es exclusiva del cuidado, fenómenos como la experiencia del padecimiento fue posible analizarlo gracias a la enfermedad crónica, situación que las enfermedades infectocontagiosas difícilmente permitían hacer ${ }^{9}$. Lo mismo acontece con la vejez, nuevas categorías sociales emergieron con el aumento de la esperanza de vida 10 .

El cuidado informal casi siempre es otorgado por la familia. De acuerdo a Brody 11, debido al incremento de la esperanza de vida y de las enfermedades crónicas, la familia se vio obligada a inventar el cuidado de largo plazo. En el mismo sentido, Ellis-Hill \& Payne 12 afirman que con el mayor número de enfermos crónicos viviendo en el hogar hubo la necesidad de construir el término de cuidado para poder incluir a la familia como un elemento más de la atención a la enfermedad en este grupo de enfermos.

\section{El cuidado en el hogar: la autoatención a la enfermedad crónica}

La autoatención es “....la realización de una serie de actividades orientadas directa o indirectamente a asegurar la reproducción biológica y social a partir de la unidad domésticalfamiliar..." 2 (p. 176); proceso que se da a diversos niveles: individual, familiar, de la red social o la comunidad 1 . De acuerdo con esta definición, la autoatención incluiría cualquier proceso de atención a la enfermedad encaminado a la reproducción biológica y social del enfermo y puede realizarse en cualquiera de los niveles anteriormente señalados; esto implica reconocer, teóricamente, la existencia de múltiples procesos de autoatención que coexisten y operan simultáneamente en diferentes niveles sociales. Desde esta perspectiva no habría contraargumentos para pensar en el sistema de autoatención como un proceso único. Bajo estas consideraciones es como propongo el cuidado en el hogar a los enfermos crónicos adultos y ancianos como un proceso de autoatención.

Mi punto de partida es que el cuidado en el hogar es una acción social dirigida a asegurar la reproducción biológica y social de un enfermo crónico. Los dos fines del cuidado son la satisfacción de las necesidades creadas por la presencia de la enfermedad y la de proporcionar los recursos suficientes para su supervivencia. Varias argumentaciones sustentan mi afirmación.

Ser un enfermo crónico remite, en última instancia, a la definición social de ser un sujeto dependiente. Condición social en la que un individuo es incapaz de satisfacer sus propias necesidades y de cuidar de sí mismo, por lo que es forzado a confiar en el cuidado provisto por otros 13. La enfermedad crónica produce una dependencia paulatina e irreversible de tipo orgánica y social por lo que nunca se retorna a la categoría de sujeto sano. El cuerpo orgánico pierde lentamente sus desempeños fisiológicos hasta que es incapaz de realizarlos por sí mismo; de igual manera, su cuerpo social sufre pérdidas que se objetivan, sobre todo, en la ejecución deficiente de sus roles de acuerdo a los estándares sociales y en la incapacidad de ser recíproco en el sistema de intercambios sociales. Los estudios sobre la experiencia de ser un enfermo crónico muestran los efectos producidos en su vida biográfica, social y orgánica y la aparición de necesidades de diversa índole: las ligadas al manejo de los síntomas y de los tratamientos prescritos; las económicas; las existenciales por el impacto en su vida biográfica; las afectivas; las vinculadas con las pérdidas de roles sociales y los conflictos emanados por estas pérdidas 14,15,16,17. Ante una condición de múltiples necesidades es por lo que el cuidado se vuelve indispensable en la vida de los enfermos crónicos.

Para que el cuidado sea garante de la supervivencia biológica y social del enfermo es necesario que emerja como una respuesta social a todas las necesidades derivadas del estatus de enfermo, no sólo de la enfermedad. Esta es una diferencia con el proceso de automedica- 
ción que exclusivamente se centra en resolver la situación de la enfermedad, olvidándose del sujeto que la padece. Para Kleinman 1 las dos tareas fundamentales de cualquier sistema de atención son la curación de la enfermedad que implica lograr un control efectivo de los desordenes biológicos y fisiológicos y el de atender al padecimiento que es contribuir a dar un significado personal y social a los problemas de la vida creados a partir del estatus de enfermo. Esto significa que el cuidado es una respuesta dirigida a la atención de la enfermedad crónica pero sobretodo al padecer del enfermo. Es a partir de estos rasgos fundamentales por lo que afirmo que el cuidado a los enfermos crónicos es un proceso de autoatención.

Menéndez 2 refiere tres tipos de actividades de la autoatención: las de alimentación, de limpieza e higiene y, las de curación y prevención de enfermedades, daños y problemas. Sin embargo, para que el cuidado responda al enfermo cómo un todo es necesario considerar otro tipo de actividades, de lo contrario, no se comprendería como es que responde a las necesidades sociales derivadas del estatus de enfermo. El cuidado es una acción social que produce bienes y servicios, a través de modificar los recursos materiales, sociales y simbólicos del contexto social donde vive el enfermo, para obtener, más que un producto único, una serie de medios de subsistencia materiales, sociales y simbólicos que satisfagan todas sus necesidades. Así, el cuidado incluye múltiples actividades diseminadas en varias direcciones de la vida social y personal del enfermo, que se agrupan en tres líneas de cuidado: de la enfermedad, del hogar y la biográfica. Y cada una de las líneas, a su vez, se divide en cuatro áreas. Las dos primeras líneas son las más estudiadas respecto al cuidado a ancianos y enfermos crónicos, no así la tercera.

La línea de cuidado en torno a la enfermedad resuelve las necesidades emanadas de la propia enfermedad y las de su atención. La primera área es establecer un diagnóstico que define con cierta certeza lo qué le sucede al enfermo y darle un nombre: empeoramiento, agravamiento, recuperación, crisis, descontrol, estancamiento o agonía. Diagnosticar es condición necesaria y primigenia para tomar decisiones posteriores sobre qué hacer, cuándo, cómo, dónde y por quién.

El área de manejo de las molestias busca aliviar o disminuir los malestares originados por la enfermedad o como consecuencia de los regímenes terapéuticos; evitar la aparición o el agravamiento o crisis de la enfermedad; y participar o tomar decisiones acerca de su manejo.
El área de manejo de los regímenes terapéuticos incluye la de todas sus enfermedades y todos los tratamientos prescritos por terapeutas de cualquiera de los sistemas médicos. Aquí, el cuidado se orienta a que el enfermo "cumpla" uno, parte o todos los regímenes prescritos; modifique o elimine una, algunas o todas las indicaciones prescritas de acuerdo al estado de mejoría o empeoramiento del enfermo y el grado de eficacia de la indicación terapéutica; combine o no las prescripciones provenientes de varios terapeutas o sistemas médicos; recordar la administración o administrar las indicaciones terapéuticas.

El área de atención terapéutica a la enfermedad brinda al enfermo todas las posibilidades de atención disponibles; organiza las opciones de atención de acuerdo a las necesidades del enfermo y garantiza su acceso a lo largo del tiempo; valora la calidad y eficacia de la atención brindada; y recuerda o acompaña al enfermo a sus consultas con los terapeutas e interacciona con ellos.

La línea de cuidado en torno al hogar es la construcción de un contexto propicio para la supervivencia del enfermo y al mismo tiempo para el despliegue de las otras acciones de cuidado. El área de apoyo económico cubre total o parcialmente los gastos generados por la enfermedad y su atención, la manutención del enfermo y los derivados de las modificaciones que sufre el hogar.

El área del trabajo doméstico proporciona los recursos y servicios en cuanto aseo y orden tanto del ambiente de la vivienda como de los enseres personales del enfermo. Así como la preparación de las terapias prescritas y su alimentación.

En el área de los cuidados personales las acciones substituyen las funciones y desempeños corporales del enfermo. Estas acciones incluyen, entre otras, la realización total o parcial de sus funciones de la vida diaria como comer, vestirse, asearse, moverse de un lugar a otro o transportarse fuera del hogar.

La organización de un ambiente de cuidado, la cuarta área, transforma el hogar en un "cuarto de enfermo" con la finalidad de crear condiciones físicas y simbólicas que satisfagan las necesidades del propio enfermo. Las acciones se orientan a organizar los tiempos del hogar; a crear un ambiente físico propicio para el enfermo mediante arreglos domésticos de mobiliario y espacio; crear un ambiente social favorable y; organizar el cuidado en cuanto a qué hacer y cuándo.

La línea de cuidado biográfico tiene como finalidad ayudar al enfermo a reconstruir su 
identidad. La enfermedad produce cambios corporales y efectos en la vida social que impactan la vida biográfica de los enfermos, por lo que deben reconstruir su percepción de sí mismos y de su relación con el mundo, pero incorporando la enfermedad como parte esencial de su vida 18 . Y el cuidado contribuye a estos esfuerzos del enfermo por lograrlo. Esta línea es la más invisible de las tres y la que demanda mayores esfuerzos para su realización; generalmente se le liga a la dimensión emocional y sus acciones se confunden con expresión de sentimientos de otro tipo de relaciones interpersonales, como las de parentesco.

El área de apoyo para la reconstrucción de la identidad del enfermo crea condiciones favorables para que recupere o mantenga una percepción de sí mismo como un ser autónomo y capaz de controlar su vida y a su enfermedad. Ello implica apoyar sus decisiones, dar ánimos, consuelo o apoyo espiritual y respetar su autonomía.

El área para evitar el aislamiento social son acciones dirigidas a mantener los contactos sociales del enfermo cuando ya no es capaz de mantenerlos vigentes por sí mismo. Esta tarea implica facilitar o ser mediador de los contactos con los miembros de su red social y/o mantener sus actividades sociales y recreativas a través de acompañar, conversar, invitarlo a reuniones o salidas recreativas.

El área para dar continuidad al curso de vida del enfermo tiene el fin de conservarlo socialmente vivo a través del cumpliendo de sus roles sociales. Ya sea apoyándolo o substituyendo parcial o totalmente sus funciones sociales en el nivel laboral o familiar; participando o tomando decisiones sobre el manejo de su enfermedad y el curso de su vida.

El área para la creación de una relación de cuidado con el enfermo implica incorporar el cuidado a la vida biográfica y social, tanto del enfermo como de quien lo cuida, lo que da paso a una nueva relación interpersonal con el enfermo, diferente al resto de sus relaciones sociales y de parentesco. Lo que implica, para ambos, incorporar el cuidado a sus vidas y trabajarlo en términos de convertirlo en un elemento de la cotidianidad. La característica de esta relación es que quien cuida se convierte en confidente, co-partícipe y la persona más cercana al enfermo al compartir y solidarizarse cotidianamente con él o ella en su experiencia como enfermo(a) en los cambios en su vida, así como con sus emociones y pensamientos.
Las acciones de cuidado y sus particularidades de funcionamiento

El cuidado posee seis características. La primera, el fin de una acción cambia de acuerdo a las circunstancias. El "no decirle algo" al enfermo varía el fin y de acuerdo a sus condiciones. Si está en una fase estable, no comentarle problemas familiares tiene un sentido preventivo. En cambio, si está en una fase de deterioro, no decirle la gravedad de su estado es para proteger su identidad.

La segunda, una acción no es exclusiva de una línea de cuidado, ya que puede pertenecer simultáneamente a varias áreas y líneas de cuidado. "Dar tés" (infusiones) al enfermo, es una acción de la línea del hogar del área de trabajo doméstico por su preparación; al mismo tiempo se ubica en la línea de la enfermedad como una medida para aliviar molestias - del área de manejo de las molestias - y como parte de la búsqueda de opciones de atención terapéutica.

La tercera característica, el cuidado es un continuum. Conforme el enfermo pierde autonomía, las acciones lo substituyen cada vez más. En el aseo personal, primero, se le coloca una silla para que se bañe sentado, después, se le ayuda a enjabonarse; más adelante, se le baña en la cama.

La cuarta característica, hay acciones centrales y periféricas. Estas últimas crean las condiciones propicias o ponen al alcance los recursos necesarios para las acciones centrales. Transportarlo a los centros de urgencias es una acción previa y necesaria para lograr que reciba atención médica. En cambio, las acciones centrales resuelven directamente las necesidades del enfermo. Por ejemplo dar consuelo.

La quinta característica, acciones procedentes de varias líneas y áreas forman una "unidad de cuidado" porque tienen un arreglo en tiempo y espacio secuencial y no son independientes. Una "unidad de cuidado" durante una crisis consistiría en identificarla, decidirse por una atención médica de urgencias y trasladarlo al hospital. En esta unidad las acciones pertenecen a tres áreas: establecimiento de un diagnóstico, atención terapéutica a la enfermedad y cuidados personales.

La última es la "invisibilidad" de ciertas acciones como cuidado. La no visibilidad es porque originalmente pertenecen a otras esferas de la vida cotidiana y el cuidado se las apropia. El trabajo doméstico es un ejemplo claro de esta situación. La preparación de los alimentos para la familia es una actividad previa a la enfermedad, cuando la preparación se ajusta a las indicaciones de un régimen dietético se convierte en una acción de cuidado. 


\section{La permanencia del cuidado}

Se afirma que la autoatención es la primera actividad terapéutica ante la enfermedad por lo que está presente, invariablemente, al inicio de la carrera del enfermo 1,2. En el cuidado a los enfermos crónicos no sucede así, ya que está presente no sólo al inicio de la trayectoria del padecimiento y de la carrera del enfermo, sino a lo largo de ambas situaciones. Su permanencia deriva de una característica central de la enfermedad crónica: su incurabilidad. Además, dicha permanencia no es estática, sino dinámica. El cuidado se transforma y responde a las necesidades cambiantes del enfermo conforme la enfermedad avanza y afecta más y más la vida orgánica, personal, familiar y social del enfermo 19.

En un inicio, el cuidado son ayudas específicas por breves períodos de tiempo que se otorgan cuando el enfermo demanda ayuda. Las acciones se ubican en el nivel más bajo de la sustitución de las funciones corporales y sociales del enfermo, razón por la que se realizan sólo una parte de ellas en cada una de las líneas de cuidado. Posteriormente, y con un mayor deterioro orgánico y social del enfermo, el cuidado se convierte en una práctica social de cuidado, se establecen "rutinas de cuidado" y se llevan a cabo todas las acciones de las tres líneas de cuidado. Durante la agonía, cuando poco se puede hacer por aliviar el sufrimiento del enfermo, el cuidado entra a una fase de retiro paulatino, se reduce a unas cuantas acciones y se da un proceso de separación simbólica entre quien cuida y el enfermo. La terminación del cuidado se da con la muerte del enfermo, es decir, el cuidado no desaparece con la curación o alivio de la enfermedad como sucede con la automedicación, sino con la muerte.

\section{¿Quién cuida a los enfermos crónicos?}

De acuerdo con Menéndez 20 (p. 139) “...no es la familia sino la mujer la encargada de asumir las actividades referentes a la salud/enfermedad/atención. Esto se pone de relieve, frecuentemente sin demasiada reflexión metodológica, en el trabajo clínico, en el trabajo epidemiológico y socioantropológico". La autoatención no está exenta de esta feminización. Los estudios muestran como la madre/ esposa es la responsable de la autoatención de los niños y mujeres con problemas reproductivos, y en menor medida, las abuelas con respecto a los niños. En el cuidado en el hogar a enfermos crónicos adultos y ancianos se repite el mismo fenómeno, son mujeres quienes asumen el cuidado pero ya no a partir del parentesco de madre/esposa, sino del de esposa e hija. Un hecho reiteradamente reportado en la literatura anglosajona $21,22,23$ y que también comienza a documentarse para América Latina y España 24,25,26,27,28.

La presencia de la esposa e hija como cuidadoras de enfermos adultos y ancianos se comprende a partir del ciclo de vida familiar. La opción ideal para los niños es la vía generacional ascendente en primera e incluso segunda generación, es decir, la madre o la abuela. Los niños no han procreado por lo que es imposible pensar en una generación descendente. Optar por la madre y la abuela es posible porque la familia del niño se encuentra en fase de expansión. Las familias de los enfermos crónicos adultos y ancianos, por el contrario, se encuentran en las últimas fases del ciclo de vida: la de dispersión o la de retiro y muerte. Los parentescos disponibles, entonces, son la esposa o las hijas, que pertenecen a la misma generación o la descendiente pero ya no a la ascendente. La esposa es un recurso, siempre y cuando no requiera ella misma de cuidados por estar enferma. La otra opción son las hijas, como adultas pueden asumir esta responsabilidad plenamente. Las abuelas desaparecen de la escena como cuidadoras por el simple hecho de que casi siempre son ellas quienes requieren de ser cuidadas, en este caso abandonan el rol de cuidadoras y se convierten en las madres enfermas que son cuidadas por sus hijas.

\section{Sobre las futuras investigaciones}

No todos los enfermos son atendidos por terapeutas, los estudios revelan que una mínima cantidad de los episodios mórbidos llegan a alguno de ellos y que la mayoría de los enfermos se atienden y son atendidos por sus familiares. En países desarrollados, alrededor de $20 \%$ de los episodios de enfermedad llegan a los terapeutas, el resto se atienden en el hogar 29. Y es que la mayoría de los vastos esfuerzos por mantener la salud como por atender la enfermedad se dan al interior del hogar y son proporcionados por los propios enfermos, su familia o los miembros más cercanos de su red social. Lo anterior deriva de que realmente los individuos dedican poco tiempo de su vida a ser pacientes y son casi todo el tiempo, enfermos 17.

Negar o estigmatizar los saberes y prácticas ante la enfermedad por parte de los legos sólo oculta la riqueza y variedad de respuestas que se generan a nivel de la autoatención. Indagar sobre la misma es fundamental para entender qué hacen los sujetos enfermos y por qué hacen o dejan de hacer determinadas prácticas a pesar de los esfuerzos por influir en ellas. La in- 
comprensión de este nivel de atención a la enfermedad ha llevado a convertir en invisible un fenómeno bastante común en cualquier tipo de sociedad. De ahí la importancia de un mayor número de investigaciones empíricas que documenten su existencia, pero también de esfuerzos conceptuales por explicar su funcionamiento y características.

Uno de los problemas centrales en el estudio del tema es la preeminencia del concepto de enfermedad como punto de partida o eje analítico. Cualquier indagación sobre la atención a partir de la noción de enfermedad lleva a olvidar las influencias de los factores sociales y culturales, tanto en la definición de la enfermedad como en las respuestas sociales para su atención. Los estudios sobre la autoatención no sólo deben partir de la noción de enfermedad, sino también de la de padecimiento, para dar cuenta de prácticas más allá de las ligadas a la automedicación. Lo que se privilegia con una $u$ otra mirada tiene consecuencias en el tipo de acciones que se incluyen/excluyen en el momento del análisis de la autoatención. La automedicación excluye todo tipo de acción vinculada con el estatus de enfermo y sólo privilegia las concernientes a las terapéuticas, por lo que deja fuera del análisis lo relativo al cuidado del trabajo biográfico.

Segundo, es necesario analizar los diversos niveles de autoatención. No sólo el enfermo realiza este tipo de prácticas, sino igualmente la familia, la red social y la comunidad; visualizar solamente alguno de ellos nos lleva a concluir que la autoatención es un proceso único y no plural. El pluralismo es un rasgo de los sistemas de atención a la enfermedad. Dado este rasgo, la autoatención no tendría porqué ser diferente.
Así, el cuidado no es el único, a nivel individual está el autocuidado y a nivel comunitario, el cuidado formal. Ambos, junto con el cuidado en el hogar constituyen elementos del proceso de cuidado a los enfermos crónicos como una forma de autoatención. Indagar sobre cada uno de ellos es una tarea impostergable. Identificar las prácticas propias de autoatención en cada uno de los niveles, la variedad que existe al interior de cada una de ellas, las relaciones de las prácticas de un nivel con los otros y los momentos en que se unifican, deberían ser temas de la futura agenda de investigación en esta área.

Tercero, reconocer que la autoatención no es una práctica que aparece sólo al inicio de la carrera del enfermo. La enfermedad crónica permitió visualizar cómo los enfermos entran y salen de uno y otro sistema de atención de acuerdo a sus necesidades; ahora los enfermos recurren a la autoatención no sólo antes del diagnóstico, sino también después del mismo. Lo que argumento es que los procesos de autoatención son permanentes a lo largo de la carrera del enfermo y no sólo en sus inicios, el asunto es identificarlos.

Finalmente, la cuestión de los saberes en torno a la autoatención y de sus diversos procesos es un asunto primordial. Saberes en torno a cómo se explica el origen, desarrollo y curación de la enfermedad, pero también en cómo se percibe, nombra, explica, evalúa y se responde a ella. Las prácticas de autoatención requieren de estos saberes para decidir qué, cómo y cuándo actuar. De igual forma, requerimos saber cómo interactúan estos saberes con los saberes de la biomedicina y las medicinas alternativas, ya sea en sus transacciones, conflictos, integración o subordinación.

\section{Resumen}

Este trabajo destaca la importancia del cuidado en el hogar a los enfermos crónicos adultos y ancianos como un componente del sistema de atención. La tesis central es que el cuidado en el hogar deber ser conceptualizado como un proceso de autoatención. Su finalidad es garantizar la supervivencia orgánica y social del enfermo, y va más allá de la atención a la enfermedad. El cuidado está integrado por tres líneas de cuidado: de la enfermedad, del hogar y la biográfica. El mismo está presente a lo largo de la trayectoria del padecimiento y la carrera del enfermo; y es realizado casi siempre por mujeres. La propuesta se discute en el marco del proceso de envejecimiento, los cambios epidemiológicos acaecidos a nivel mundial y la urgencia de incorporar este análisis en la agenda de investigación de los sistemas de atención a la enfermedad. 


\section{Referencias}

1. Kleinman A. Patients and healers in the context of culture. An exploration of the borderland between anthropology, medicine and psychiatry. Berkeley: University of California Press; 1980.

2. Menéndez E. Antropología médica. Orientaciones, desigualdades y transacciones. México DF: Centro de Investigaciones y Estudios Superiores en Antropología Social; 1990. (Cuadernos de la Casa Chata 179).

3. Freund P, McGuire M. Health, illness and the social body. A critical sociology. $3^{\text {rd }}$ Ed. Upper Saddle River: Prentice Hall; 1999.

4. Mirkin B, Weinberger MB. The demography of population ageing. New York: United Nations. 2000.

5. World Health Organization. The world health report 1998. Executive summary. http://www.who. int/whr/1998/whr-en.htm (accesado el 18/Oct/ 2000).

6. Cantor M. Family and community: changing roles in an aging society. Gerontologist 1991; 31:337-46.

7. Kane R, Penrod JD. In search of family caregiving policy: General considerations. In: Kane R, Penrod JD, editors. Family caregiving in an aging society. Policy perspectives. v. 5. Thousand Oaks: Sage; 1995. p. 1-14. (Family Caregiver Applications Series).

8. Pearlin LI, Zarit S. Research into informal caregiving: current perspectives and future directions. In: Zarit S, Pearlin LI, Warner-Schaie K, editors. Caregiving systems. Formal and informal helpers. Hilsdale: Lawrence Erlbaum Associates; 1993. p. 155-67.

9. Charmaz K. Discovering chronic illness: using Grounded Theory. Soc Sci Med 1990; 30:1161-72.

10. Canales A. La población en la era de la información. De la transición demográfica al proceso de envejecimiento. Estudios Demográficos Urbanos 2001; 16:485-518.

11. Brody EM. Prospects for family caregiving: response to change, continuity, and diversity. In: Kane RA, Penrod J, editors. Family caregiving in an aging society. Policy Perspectives. v. 5. Thousand Oaks: Sage Publication; 1995. p. 15-28. (Family Caregiving Applications Series).

12. Ellis-Hill C, Payne S. The future: interventions and conceptual issues. In: Payne S, Ellis-Hill C, editors. Chronic and terminal illness. New perspectives on caring and carers. New York: Oxford University Press; 2001. p. 155-66.

13. Kittay EF. Love's labor. Essays on women, equality and dependency. New York: Routledge; 1999.

14. Corbin J, Strauss A. Accompainments of chronic illness: changes in body, self, biography, and biographical time. In: Roth J, Conrad P, editors. The experience and management of chronic illness. Research in the sociology of health care. v. 6. Greenwich: Jai Press Inc.; 1987. p. 249-81.
15. Corbin J, Strauss A. Unending work and care. Managing chronic illness at home. San Francisco: Jossey-Bass Publishers; 1988.

16. Mercado F. Entre el infierno y la gloria. La experiencia de la enfermedad crónica en un barrio urbano. Guadalajara: Universidad de Guadalajara; 1996.

17. Strauss A, Corbin J, Fagerhaugh S, Glaser BG, Maines D, Suczek B, et al. Chronic illness and the quality of life. $2^{\text {nd }}$ Ed. Sant Louis: Mosby Company; 1984

18. Charmaz K. Struggling for a self: identity levels of the chronically ill. In: Roth J, Conrad P., editors. The experience and management of chronic illness. Research in the sociology of health care. vol. 6. Greenwich: Jai Press Inc.; 1987. p. 238-321.

19. Biegel D, Sales E, Schulz R. Family caregiving in chronic illness. Alzheimer's disease, cancer, heart disease, mental illness and stroke. Newbury Park: Sage Publication; 1991.

20. Menéndez E. Familia, participación social y proceso salud/enfermedad/atención. Acotaciones desde las perspectivas de la antropología médica. In: MercadoFJ, Robles L, Denman C, Escobar A, Infante C, editors. Familia, salud y sociedad. Experiencias de investigación en México. Guadalajara: Universidad de Guadalajara/Instituto $\mathrm{Na}$ cional de Salud Pública/Centro de Investigaciones y Estudios Superiores en Antropología Social/El Colegio de Sonora; 1993. p. 130-62.

21. Allen S, Goldscheider F, Ciambrone DA. Gender roles, marital intimacy, and nomination of spouse as primary caregiver. Gerontologist 1999; 39:1508

22. Lee G, Dwyer J, Coward RT. Gender differences in parent care: demographic factors and same-gender preferences. J Gerontol 1993; 48:S9-S16.

23. Stone R, Cafferata GL, Sangl J. Caregivers of the frail elderly: a national profile. Gerontologist 1987; 27:616-26.

24. Caldas C. Envelhecimiento com dependência: responsabilidades e demandas da familia. Cad Saúde Pública 2003; 19:773-81.

25. Karsch UM. Idosos dependentes: famílias e cuidadores. Cad Saúde Pública 2003; 19:861-6.

26. Robles L. El fenómeno de las cuidadoras: un efecto invisible del envejecimiento. Estudios Demográficos Urbanos 2001; 16:561-84.

27. Seira MP, Aller A, Calvo A. Morbilidad sentida y diagnosticada en cuidadores de pacientes inmovilizados de una zona de salud rural. Rev Esp Salud Pública 2002; 76:713-21.

28. Setién ML. Género y cuidados a las personas en el espacio doméstico. Inguruak 1998; 22:79-94.

Recibido el 9/Jul/2003

Versión final presentada el 28/Oct/2003

Aprobado el 22/Dic/2003 